\title{
Limited research on surgical interventions to accelerate orthodontic treatment
}

\author{
Abstracted from \\ Fleming PS, Fedorowicz Z, Johal A, El-Angbawi A, Pandis N. \\ Surgical adjunctive procedures for accelerating orthodontic treatment. \\ Cochrane Database Syst Rev 2015; Issue 6. Art. No. CD010572. DOI: 10.1002/14651858.CD010572.pub2. \\ Address for correspondence: Luisa Fernandez Mauleffinch, Managing Editor, Cochrane Oral Health Group, \\ School of Dentistry, The University of Manchester, JR Moore Building, Oxford Road, Manchester, M13 9PL, UK. \\ E-mail: luisa.fernandez@manchester.ac.uk
}

\section{Question: Can surgical procedures speed up orthodontic treatment?}

Data sources the Cochrane Oral Health Group's Trials Register, the Cochrane Central Register of Controlled Trials (CENTRAL), Medline, Embase, LILACS, metaRegister of Controlled Trials, ClinicalTrials.gov and the World Health Organisation (WHO) International Clinical Trials Registry Platform.

Study selection Randomised controlled trials (RCTs) evaluating the effect of surgical adjunctive procedures for accelerating tooth movement compared with conventional treatment (no surgical adjunctive procedure).

Data extraction and synthesis Two reviewers independently abstracted data and assessed risk of bias.

Results Four randomised trials involving 57 patients were included. All four studies had unclear risk of bias. Interventions evaluated were corticotomies to facilitate orthodontic space closure or alignment of an ectopic maxillary canine, with the effect of repeated surgical procedures assessed in one of these studies. Tooth movement was found to be slightly quicker with surgically assisted orthodontics in comparison with conventional treatment over periods of one month (MD $0.61 \mathrm{~mm} ; 95 \% \mathrm{Cl} 0.49$ to 0.72 ; $\mathrm{P}$ value $<0.001$ ) and three months (MD $2.03 \mathrm{~mm}, 95 \% \mathrm{Cl} 1.52$ to 2.54 ; P value $<0.001$ ). No information on adverse events was reported in the included studies. Conclusions This review found that there is limited research concerning the effectiveness of surgical interventions to accelerate orthodontic treatment, with no studies directly assessing our prespecified primary outcome. The available evidence is of low quality, which indicates that further research is likely to change the estimate of the effect. Based on measured outcomes in the short-term, these procedures do appear to show promise as a means of accelerating tooth movement. It is therefore possible that these procedures may prove useful; however, further prospective research comprising assessment of the entirety of treatment with longer follow-up is required to confirm any possible benefit.

This paper is based on a Cochrane Review published in the Cochrane Library 2015, issue 6 (see www.thecochranelibrary.com for information). Cochrane Reviews are regularly updated as new evidence emerges and in response to feedback, and the Cochrane Library should be consulted for the most recent version of the review.

\section{Commentary}

This Cochrane Collaboration systematic review with a meta-analysis covers a topic of recent major interest among the orthodontic community. For some prospective patients an orthodontic treatment that is shorter provides them with a faster aesthetic and functional result. Overall, given a scenario where all other influencing variables are the same, who would not want a faster treatment? As most situations in life reality is an extremely complex multifaceted problem that cannot be decided based on one single variable. Let's explore this further.

Surgical approaches as expected would come with an increased biological and financial cost. So the real question is if patients, under a proper informed consent process, would accept the extra financial burden (which in some cases may be $50-75 \%$ of the total orthodontic cost) to save a few months of orthodontic treatment time. The tipping point to answer this question is obviously dependent on the patient's values, financial resources and full comprehension of the pros and cons of such a decision. So far the biological impact of these adjunctive surgical procedures is only understood on a short-term basis. The long-term consequences are unknown.

As expected this Cochrane Collaboration systematic review is solid and methodological, and any of its drawbacks are related to the quality of the available literature. An acceptable search strategy was proposed, several steps were undertaken to minimise potential selection and analysis biases, and the conclusions seem to be properly tempered. The fact that only randomised clinical trials were included is controversial. Although for questions about treatment effects RCTs are the gold standard, there is some evidence that lower levels of evidence should not automatically be discarded. So there is a potential that some useful information may have been excluded.

At the end of the day conclusions were based on a total combined sample of 57 participants (yes no typo there!) from the four finally included RCTs. So how safe can we feel in making clinical decisions for our patients based on such a small sample for a potentially harmful surgical process? To further compound our level of uncertainty the main outcomes of this SR were not directly addressed by the available evidence. Basically only the rate of tooth movement when closing extraction spaces was assessed. Secondary outcomes were periodontal status and pain levels (no apparent differences there). In summary, areas exposed to corticotomies favoured a faster tooth movement of around $0.6 \mathrm{~mm}$ per month. So in a case in which a full premolar extraction space (assuming $7 \mathrm{~mm}$ ) is to be closed the saving 
would be equivalent to 3.5 months (assuming normal space closure rate to be around $1 \mathrm{~mm}$ ) compared to a usual seven months. So an orthodontic treatment that may have lasted 24 months will now last 20 months (16\% time savings). Assumptions can be adjusted, but the principle stays. Going back to our initial question: is a saving of four months (16\%) worth the extra biological and financial cost of the adjunctive therapy? The answer may or may not be positive and is specific to every individual situation.

To conclude: a significant amount of meaningful information is still unknown. Is the total duration of treatment, number of visits or final outcome modified significantly? We do not know. The potential impact on extraction space closure is there. The overall impact of surgically assisted adjunct procedures to accelerate orthodontic treatment is still questionable.

\section{Carlos Flores-Mir}

Division Head of Orthodontics, University of Alberta, Edmonton, Canada

Evidence-Based Dentistry (2015) 16, 88-89. doi:10.1038/sj.ebd.6401120 